Z. klin. Chem. u. klin. Biochem.

10. Jg. 1972 , S. $160-163$

\title{
Automation der enzymatischen Triglyceridbestimmung in Serumproben
}

\author{
Von F. H. Schmidt, Gisela Schmidt, Katharina von Dahl ind H. Stork \\ Aus den Forschungslaboratorien der Firma Boebringer Mannbeim GmbH, Mannheím
}

(Eingegangen am 4. Oktober 1971)

\begin{abstract}
Eine relativ rasche und sehr zuverlässige Adaptation der enzymatischen Triglyceridbestimmung im Serum auf einen Automaten wird beschrieben. Im Prinzip wird wie bei der manuellen Technik nach vollständiger Hydrolyse Glycerin mit ATP und Glycerokinase umgesetzt und mit den Hilfsenzymen Pyruvatkinase und Lactatdehydrogenase im optischen Test der NADH-Verbrauch gemessen. Die Technik der beschriebenen Modifikation basiert auf einer Kombination von automatisierten Pipettier- und Verdünnungsschritten mit alkalischer Hydrolyse der Proben und Messung der Glycerinkonzentration im Enzymautomaten LKB 8600. Dabei wird vorgefertigtes Analysengemisch verwendet und Glycerokinase als Startenzym benutzt. Das Verfahren erlaubt die Durchführung von mindestens 30 Bestimmungen pro Stunde, die durch Erhöhung der Enzymaktivitäten auf 60 heraufgesetzt werden kann. Die Messung kann wartungsfrei über Nacht erfolgen. Die Bestimmung ist spezifisch für Glycerin und damit für Glyceride; sie ist mit einer hohen Präzision und einer guten Richtigkeit ausgestattet.
\end{abstract}

\section{Automation of the enzymic determination of triglycerides in serum samples}

The enzymic determination of triglycerides has been adapted to an automated method, which is relatively quick and very reliable. As in the manual method, glycerol is produced by complete hydrolysis and then reacted with ATP and glycerokinase. This system is coupled with the auxiliary enzymes pyruvate kinase and lactate dehydrogenase and the consumption of NADH is measured spectrophotometrically.

The present modification is based on automation of the pipetting and dilution steps, with alkaline hydrolysis of the samples and measurement of the glycerol concentration in the enzyme autoanalyser LKB 8600. The reagent mixtures are preprepared and glycerokinase is the starter enzyme. The method can be run overnight, without supervision, at the rate of at least 30 and if necessary 60 determinations per hour. It is specific, very precise and the accuracy is good.

Die Bestimmung der Triglyceride im Blutserum gewinnt sowohl als "Screening" in der Vorsorgeuntersuchung als auch für Diagnose und Therapiekontrolle von Hyperlipoproteinämien an erheblicher Bedeutung; die Zahl der Analysen ist daher in ständiger Zunahme begriffen.

Aus diesem Grund scheint es erforderlich, die wegen ihrer Zuverlässigkeit und Eleganz ausgezeichnete enzymatische Methode $(1,7)$ auf Analysenautomaten zu adaptieren, um hierdurch größere Zahlen von Analysen in relativ kurzer Zeit durchsetzen zu können. Zwar ist 1964 die kolorimetrische Technik von HANDEL und Zilversmir (2) teilautomatisiert beschrieben worden, doch ist - bedingt durch zahlreiche Vorbereitungsschritte - zur Abtrennung unspezifischer Chromogene eine umständliche Handhabung notwendig und eine nur geringe Spezifität vorhanden (6).

Das gilt in gleicher Weise für die Bestimmung der Triglyceride mit fluorimetrischer Technik (4). Ein kürzlich beschriebenes Verfahren für freies Glycerin zeigt hohe Instabilität des Reagenziengemisches (3).

Mit Hilfe eines von H. KLOTzsch und S. KrotzsCH (5) für den Enzymautomaten LKB 8600 entwickelten Verfahrens haben wir durch Kombination mit einem Pipettierautomaten und nach einigen geringfügigen Modifikationen eine brauchbare Technik für die Durchführung von 30 Analysen pro Stunde ausgearbeitet; die Zahl der Analysen kann auf 60 erhöht werden, wenn die Glycerokinaseaktivität im Ansatz verdoppelt wird.
Hierbei muß allerdings ein Fehlbetrag von etwa 7\%, der sich durch eine apparativ bedingte Verzögerung des Mẹvorganges (etwa 4 Sek.) ergibt, berücksichtigt werden. Bei längeren Meßzeiten ist die Korrektur zu vernachlässigen.

Mit diesem neuen Verfahren, das für Serum einen VK in der Serie von im Mittel 3,2\%; von Tag zu Tag mit $2,8 \%$ und im Vergleich zur manuellen Technik einen Korrelationskoeffizienten $r$ von 0,99 bei $n=249$ aufweist, haben wir inzwischen mehr als 10000 Analysen durchgeführt.

Der besondere Vorteil des mit Ausnahme des Hydrolyseschrittes automatisierten Verfahrens liegt $u$. a. auch darin, da $\beta$ die Analyse vorbereiteter Proben ohne Wartung des Gerätes über Nacht laufen kann und jeweils am folgenden Morgen die Auswertung der geschriebenen Ergebnisse gestattet, was in der Regel höchstens 60 Minuten in Anspruch nimmt.

Die Technik wird nachstehend beschrieben.

\section{Methodik}

Hydrolyse

Die Verseifung der Triglyceride im Serum wird mit 0,5N äthanol. $\mathrm{KOH}$ (glycerinfrei) in Reaktionsgefäßen aus Kunststoff oder Glas durchgeführt.

Man pipettiert (ggf. automatisch)

$\begin{array}{ll}\text { Serum } & 0,1 \mathrm{ml} \\ \text { äthanol. } \mathrm{KOH} & 0,25 \mathrm{ml} \\ 0,5 \mathrm{~N} & \end{array}$

schüttelt durch und hydrolysiert bei $55-70^{\circ} 20 \mathrm{Min}$. lang. 
Vorbereitung für die enzymatische Bestimmung Nach Abkühlen des Hydrolysates werden $0,5 \mathrm{ml} \mathrm{MgSO}_{4}$-Lösung $(0,15 \mathrm{M})$ hinzugegeben, geschüttelt und zentrifugiert. Die Lagerfähigkeit der so vorbereiteten Proben beträgt im Kühlschrank mindestens 8 Tage.

\section{Reagenzien für den enzymatischen Test \\ Gebrauclsslösung $A^{1}$ )}

Die Gebrauchslösung setzt sich aus den nachstehenden Reagenzien zusammen, die in ihren jeweiligen Endkonzentrationen in der Lösung aufgeführt sind

$\begin{array}{lll}\text { Triäthanolamin-Puffer, } \mathrm{pH} \sim 7,6 & & 0,1 \mathrm{M} \\ \mathrm{MgSO}_{4} & & 4 \mathrm{mM} \\ \mathrm{NADH} & \text { etwa } & 0,2 \mathrm{mM} \\ \text { ATP } & \text { etwa } & 1,3 \mathrm{mM} \\ \text { Phosphoenolpyruvat } & \text { etwa } & 0,5 \mathrm{mM} \\ \text { Lactatdehydrogenase } & \text { etwa } & 4 \mathrm{U} / \mathrm{ml} \\ \text { Pyruvatkinase } & \text { etwa } 1 \mathrm{U} / \mathrm{ml}\end{array}$

Um eine Arbeitslösung - ausreichend für etwa 100 Bestimmungen - anzusetzen, wiegt man die Substanzen entsprechend den nachfolgend beschriebenen Mengen ab. Man geht dabei so vor, daß man zunächst den Puffer herstellt (Substanz 1, 2, 3 in $150 \mathrm{ml} \mathrm{H}_{2} \mathrm{O}$ lösen) und danach die restlichen Substanzen 4-7 hinzugibt. Die so hergestellte Lösung ist bei $4^{\circ}$ mindestens 2 Tage haltbar.
1. Triäthanolamin-Hydrochlorid $2800 \mathrm{mg}$
2. $\mathrm{Na}_{2} \mathrm{CO}_{3} \quad 500 \mathrm{mg}$
3. $\mathrm{MgSO}_{4} \cdot 7 \mathrm{H}_{2} \mathrm{O} \quad 150 \mathrm{mg}$
4. $\mathrm{NADH}-\mathrm{Na}_{2} \mathrm{H}_{2} \quad 30 \mathrm{mg}$
5. ATP $-\mathrm{Na}_{2} \mathrm{H}_{2} \cdot 3 \mathrm{H}_{2} \mathrm{O} \quad 120 \mathrm{mg}$
6. Phosphoenolpyruvat $\cdot \mathrm{H}_{2} \mathrm{O} \quad 35 \mathrm{mg}$
7. Pyruvatkinase (EC 2.7.1.40)/
Lactatdehydrogenase (EC 1.1.1.27)
$4 \mathrm{mg} / \mathrm{ml}$
$1 \mathrm{ml}$

\section{Gebrauchslösung $B^{2}$ )}

Gebrauchslösung B enthält das Enzym Glycerokinase (EC 2.7.1.30) in einer Konzentration von $100 \mu \mathrm{g} / \mathrm{ml}$ bei einer spezifischen Aktivität von $85 \mathrm{U} / \mathrm{mg}$ in $2 \mathrm{M}\left(\mathrm{NH}_{4}\right)_{2} \mathrm{SO}_{4}$ suspendiert. Hiervon werden pro Test $50 \mu \mathrm{l}$ eingesetzt, so daß $5 \mu \mathrm{g}$ Enzym mit einer Aktivität von $0,4 \mathrm{U}$ benötigt werden.

Käufliches Enzym in der Konzentration $5 \mathrm{mg} / \mathrm{ml}$ wird mit $2 \mathrm{M}$ Ammoniumsulfat 1:50 verdünnt, wobei zweckmäßigerweise der jeweilige Tagesbedarf hergestellt wird, $z$. B. verdünnt man $0,2 \mathrm{ml}$ der Suspension auf $10 \mathrm{ml}$.

\section{Messung}

In die mit Einwegküvetten bestückten Küvettenständer des Enzymautomaten LKB 8600 werden die Proben mit Hilfe der Dilumatik pipettiert. Für den Tagesbedarf bzw. für eine Magazinfüllung (100 Proben) müssen 2 Reagenzienleerwerte angesetzt werden. Es empfiehlt sich, zur Analysenkontrolle (Enzymtest) jeweils einen Standard mit anzusetzen (z. B. Precinorm S, das etwa $20 \mathrm{mg} / 1$ freies Glycerin enthält.)

\section{Apparaturen}

\section{Pipettierautomat Dilumatik ${ }^{3}$ )}

Für die benötigten Volumina von $0,05 \mathrm{ml}$ Probe und $1 \mathrm{ml}$ Reagenz setzt man in die linke Position des Pipettierautomaten (,Ansaugen") eine $0,25 \mathrm{ml}$ Bürette, in die rechte Position (,Ver-

1) Es kann auch die Triglycerid-Testcombination TNAA 15989 der Firma Boehringer Mannheim verwendet werden. Der Inhalt der Flasche 1 wird mit $150 \mathrm{ml}$ dest. Wasser aufgefüllt. Darin wird der Inhalt det drei Flaschen 2 und ebenso die Enzymsuspension aus Flasche 3 gelöst.

2) Aus der Triglycerid-Testcombination TNAA 15989 der Firma Boehringer Mannheim kann Flasche 4 nach Verdünnen 1:10 mit Ammoniumsulfatlösung (2M) verwendet werden.

3) Firma Braun, Melsungen/Deutschland. dünnen") eine $2,0 \mathrm{ml}$ Bürette ein. Beide Büretten werden nacheinander mehrfach mit Gebrauchslösung A durchgespült. Danach werden die gewünschten Volumina eingestellt, und zwar für:

$$
\begin{array}{ll}
\text { „Ansaugen": } & 0,05 \mathrm{ml} \text { (Probc) } \\
\text { "Verdünnen“: } & 1,0 \mathrm{ml} \text { (Lösung A). }
\end{array}
$$

\section{Enzymautomat LKB $8600^{4}$ )}

Der Enzymanalyzer LKB 8600 wird für die Triglyceridbestimmung zur Endwertbestimmung eingesetzt. Vor Beginn der Analyse ist in der nachstehend angegebenen Reihenfolge der Apparat zu bedienen:

a) Nullpunkt-Einstellung

b) Hintergrund-Einstellung (0.6)

c) Schalter auf "Decrease" stellen

d) Schalter auf „Range $0,2^{\text {“ }}$ stellen

e) Schalter „Meas. Time“ auf 2 Min. stellen

f) Füllen der Pumpe mit Lösung B und auf $50 \mu \mathrm{l}$ einstellen

g) Magazin mit Küvettenständern füllen und Apparat starten.

Genauere Anweisungen sind aus den Betriebsvorschriften der Geräte zu entnehmen.

Auswertung der Analysen

Mit Hilfe der Auswertungsschablone wird $\Delta \mathrm{E}$ ermittelt; der Reagenzienblindwert wird abgezogen.

$\Delta E_{\text {korr. }} \cdot F=g$ Triglyceride/l Serum.

Berechnung des Faktors F:

Aus der allgemeinen Formel

$$
\frac{\mathrm{MG}}{\varepsilon \cdot \mathrm{d}} \cdot \frac{\mathrm{GV} \cdot 1000}{\mathrm{AV}}
$$

leitet sich der Faktor $F=26,6$ ab.

Es wird berechnet aus dem Molgewicht (MG) des Trioleins von 885 , einer Küvettenschichtdicke von $1 \mathrm{~cm}$, dem Extinktionskocffizienten $\varepsilon$ für $\mathrm{NADH}$ von $6,22 \cdot 10^{6} \mathrm{~cm}^{2} / \mathrm{Mol}$ bei $340 \mathrm{~nm}$ sowie der in den Test eingesetzten Meß- und Hydrolysatvolumina von 1,1 bzw. $0,85 \mathrm{ml}(\mathrm{GV})$ und den Probenvolumina von $0,05 \mathrm{ml}$ Hydrolysat und $0,1 \mathrm{ml}$ Serum (AV).

\section{Hinveise}

Erfassungsgrenze bei unverdünnten Seren entsprechend der Extinktionsskala des Schreibers bei $100 \mathrm{mV}: \Delta \mathrm{E}$ von 0,01 bis 0,2 entsprechend $\sim 0,25-5,0 \mathrm{~g} / 1$ Triglycerid.

Lipämische Seren müssen 1:10 mit physiol. $\mathrm{NaCl}$ verdünnt werden. Entsprechend ist der Faktor mit $10 \mathrm{zu}$ multiplizieren.

Auf Korrektur des freien Glycerins bzw. der Mono- und Diglyceride kann wegen der geringen Konzentration im Serum verzichtet werden.

Ein Reagenzienblindwert ist täglich zu erstellen - nach unseren Erfahrungen beträgt $\Delta \mathrm{E}$ hierfür 0,005 bis 0,012 . Die Berechnung mit Hilfe einer Standardlösung ist nicht zu empfehlen; eine solche Lösung (Precinorm S) sollte aber täglich einmal mitgeführt werden, um die Aktivität der Enzyme zu überwachen.

\section{Ergebnisse}

Der Arbeitsablauf unterteilt sich in zwei Pipettiervorgänge mit dem Pipettierautomaten Dilumatik sowie in die Vorbereitung und Messung der Proben im LKBAnalysenautomaten 8600. Für die Pipettierung der Proben hat sich das Verhältnis $0,1 \mathrm{ml}$ Serum: $0,25 \mathrm{ml}$ äthanol. $\mathrm{KOH}$; für die Pipettierung des Hydrolysats nach $\mathrm{MgSO}_{4}$-Zugabe $0,05 \mathrm{ml}: 1 \mathrm{ml}$ Arbeitslösung A als zweckmäßig erwiesen, so daß im Endeffekt $\sigma \mu \mathrm{l}$ Serum in die Analyse gelangen. Das Gemisch wird

4) LKB-Produkter AB Stockholm/Schweden (Vertrieb f. Deutschland: Colora Meßtechnik GmbJ, Lorch/Württemberg). 
zur Analyse automatisch in Einweg-Küvetten des LKBMagazins gefüllt. Mit dieser Anordnung können pro Stunde etwa 60 Proben vorbereitet und 30 gemessen und registriert werden. Die Meßgenauigkeit der Bestimmung wird anhand einer 6-fach-Bestimmung in einem pathologisch veränderten Serum in Abbildung 1 demonstriert. Die Werte wurden nach 1:10-Verdünnung der Serumprobe bestimmt. Aus der Abbildung ersieht man die gute Reproduzierbarkeit der Testergebnisse. Die Richtigkeit des Meßvorganges wurde mit einem glycerinhaltigen Kontrollserum überprüft. Einem 'Soll' von $20,7 \mathrm{mg} / 1$ steht bei 8 Parallelbestimmungen ein 'Ist' von $21,4 \pm 0,5$ gegenüber.

Ein Vergleich der manuellen mit der Automatentechnik anhand von 249 Serumproben bei Patienten und Gesunden wird regressionsanalytisch beschrieben. Der Korrelationskoeffizient beträgt 0,99 . Die erhaltene Regressionsgerade lautet: $Y=0,943 x+56,1$. Die Gerade verläuft nahezu unter einem Winkel von $45^{\circ}$ und schneidet die Y-Achse bei $+56,1 \mathrm{mg} / \mathrm{l}$. Ein ähnlich gutes Resultat ist vorhanden, wenn unterschiedlich

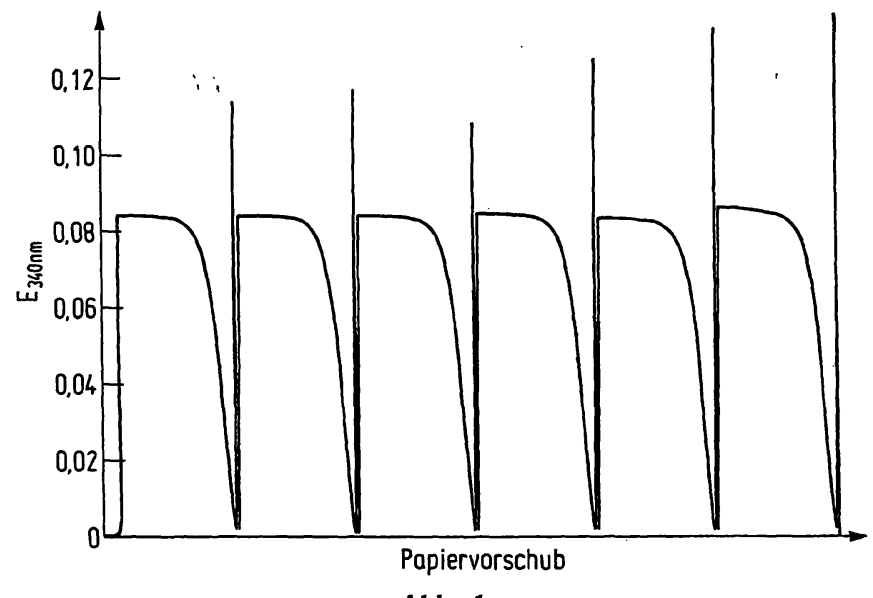

Abb. 1

Mehrfach-Bestimmung einer Serumprobe mit 10,30 g/l Triglyceride nach Verdünnung der Probe $1: 10$ und Einsetzen von 0,1 ml Hydrollysat in den Test; registriert im Automaten LKB 8600

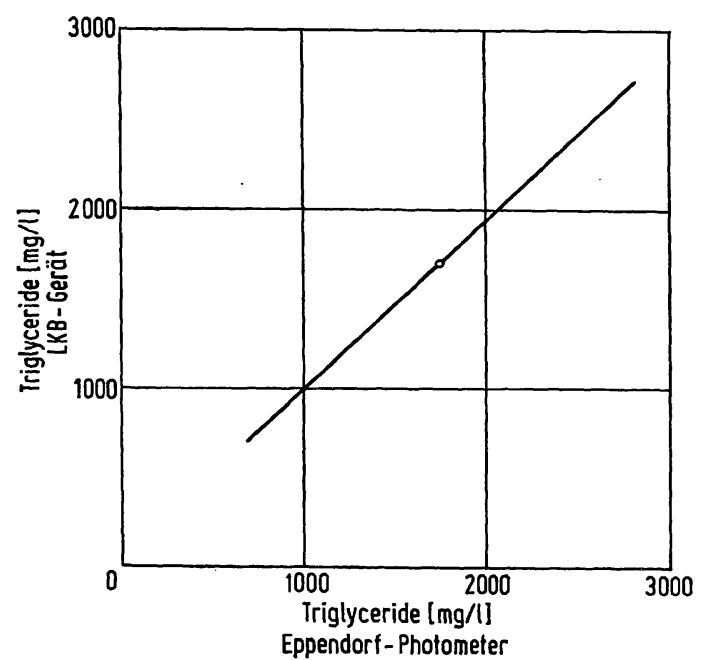

Abb. 2

Vergleich der Triglyceridbestimmungen im Serum von Patienten und gesunden Menschen $(n=249)$ zwischen enzymatischer Technik manuell, und der enzymatischen Technik mittels Analysenautomaten (LKB Uitrolab. System). Totalvergleich

$$
Y=0,943 x+56,1 ; r=0,99
$$

ausgebildete und trainierte techn. Assistentinnen die Vergleichsuntersuchungen durchführten. Untersucher I - gut trainiert - zeigt mit einem $I$ von 0,99 gegenüber Untersucher III - untrainiert - mit einem $r$ von 0,975 nur geringe Unterschiede (s. Tab. 1).

Hinsichtlich der Präzision ist der Automat der manuellen Technik überlegen, da die jeweiligen Variationskoeffizienten VK kleinere Zahlen aufweisen. Die gute Reproduzierbarkeit gilt sowohl für die Serie als auch von Tag zu Tag. Der Ermittlung des VK von 2,8\% von Tag zu Tag lagen 10 Bestimmungen an 10 verschiedenen Tagen mit einem Serum $(1,85 \mathrm{~g} / \mathrm{l}) \mathrm{zu}-$ grunde. Recovery-Untersuchungen bewegten sich bei allen Untersuchern um etwa $100 \%$, d. h., die Richtigkeit des Verfahrens ist ebenfalls sehr gut. Einzelheiten der statistischen Analysen sind in Tabelle 1 und 2 mitgeteilt.

In Abbildung 3 ist ein "Original-Tracing“ von 6 Triglyceridbestimmungen aus Serumproben des Menschen - wie sie im laufenden Betrieb anfallen - dargestellt. Die höchste hier gemessene Konzentration

Tab. 1

Statistische Auswertung von Mehrfachbestimmungen der Triglyceride im Serum

a) manuell b) automatisch

verschiedener Untersucher

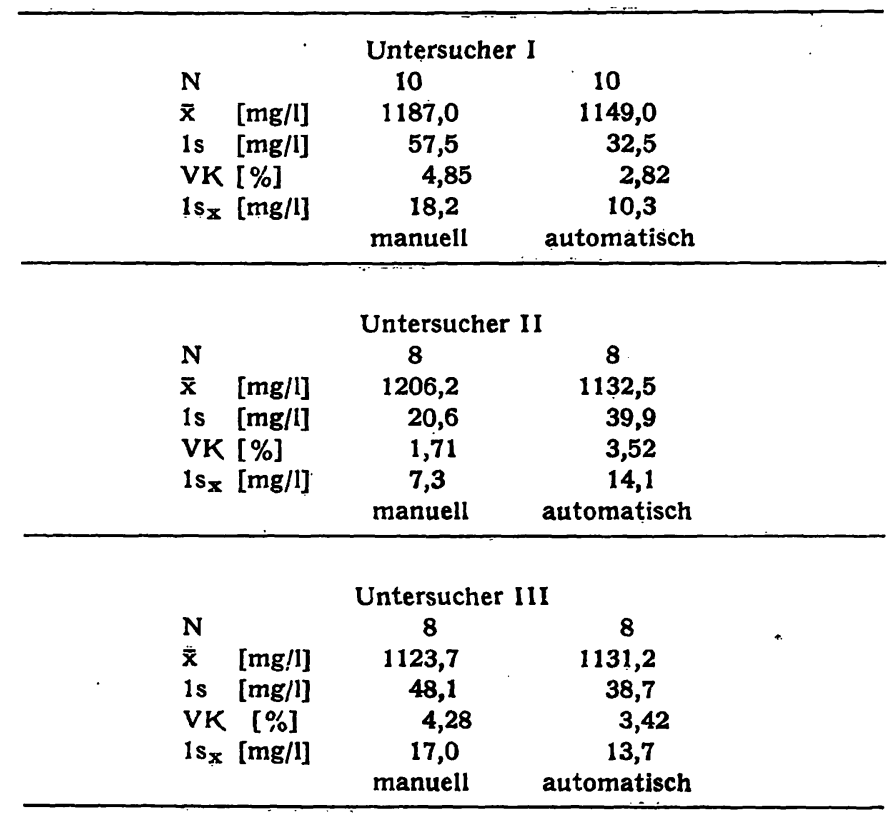

Tab. 2

Regressionsanalyse der automatisierten (y) und manuellen (x) Triglyceridbestimmung. Ergebnisse von 3 Untersuchern Errechnet wurden:

Mittelwerte $(\overline{\mathbf{x}}$ und $\bar{y})$. Standardabweichung $\mathbf{s}_{\mathbf{x}}$ und $s_{\mathbf{y}}$; Korrelationskoeffizient $r$; Ordinaten-Schnittpunkt $A$ und $M a \beta$ für Steilheit der Geraden $B$. Daraus ergibt sich für $\mathbf{Y}=B \mathbf{X}+\mathbf{A}$

\begin{tabular}{lcccc}
\hline & & Untersucher I & Untersucher II & Untersucher III \\
\hline $\mathrm{N}$ & & 174 & 37 & 38 \\
$\overline{\mathbf{x}}$ & {$[\mathrm{mg} / 1]$} & 1847,1 & 1392,4 & 1702,4 \\
Is & {$[\mathrm{mg} / 1]$} & 1105,4 & 598,3 & $904, \overline{7}$ \\
$\overline{\mathbf{y}}$ & {$[\mathrm{mg} / 1]$} & 1772,1 & 1391,1 & 1769,0 \\
Is & {$[\mathrm{mg} / \mathrm{l}]$} & 1048,1 & 595,0 & 892,4 \\
$\mathrm{r}$ & & 0,99 & 0,97 & 0,99 \\
$\mathrm{~A}$ & & 34,0 & 41,8 & 98,9 \\
$\mathrm{~B}$ & & 9,1 & 9,7 & 9,8 \\
\hline
\end{tabular}




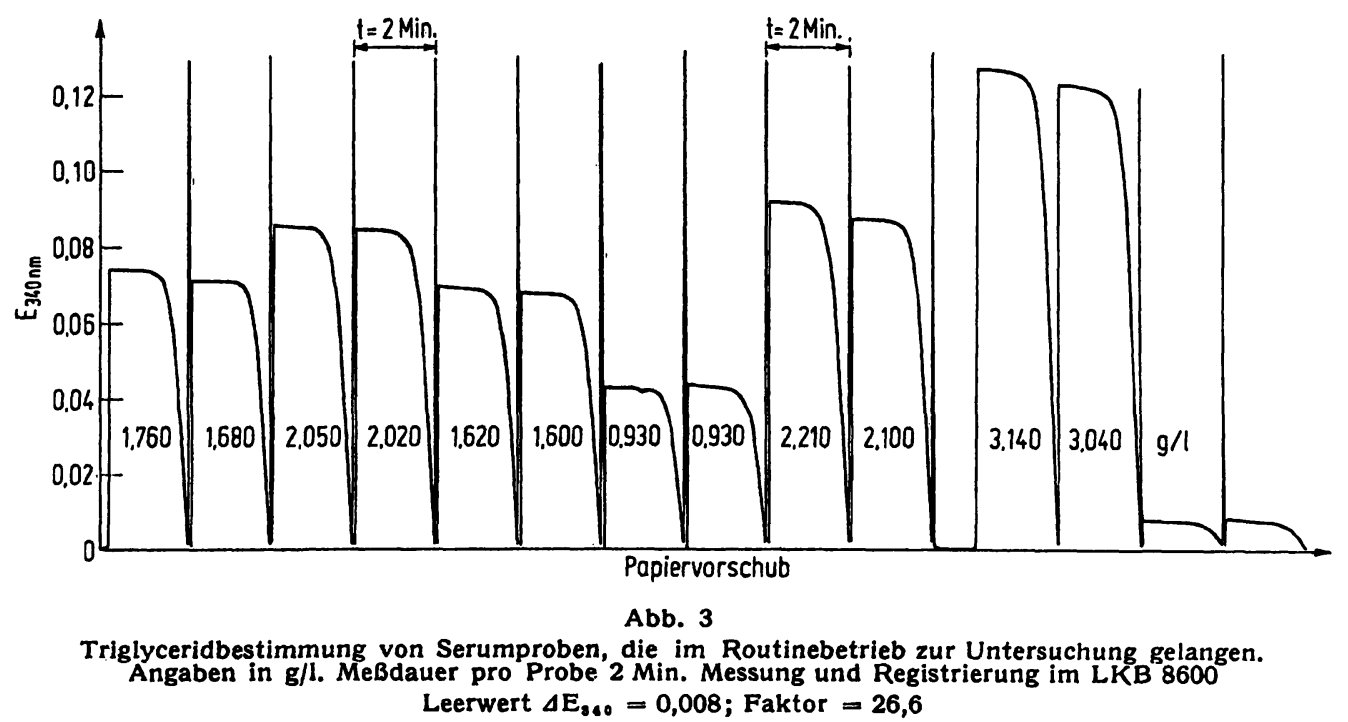

beträgt 3,14 g/l. Aus der Abbildung ist ersichtlich, daß eine maximale Konzentration an Triglyceriden bis $5,0 \mathrm{~g} / 1$ unverdünnt meßbar ist. Höhere Konzentrationen sind am maximalen Schreiberausschlag leicht $\mathrm{zu}$ erkennen und nach Verdünnen der Probe (s. Methodik) $\mathrm{zu}$ wiederholen. Bei Werten unter $0,50 \mathrm{~g} / 1$ empfiehlt es sich, das doppelte Volumen an Hydrolysat $(0,1 \mathrm{ml})$ einzusetzen.

Bei der techn. Bearbeitung und Auswertung der Ergebnisse waren weiterhin die folgenden Mitarbeiter beteiligt:

Frau E. Vondenhagen, Frau P. Heidrich, Frl. Chr. Brindley, Herr R. NEUDECKER.

\section{Literatur}

1. Eggstein, M. und F. H. Kreutz, Klin. Wschr. 44, 262 (1966). -

2. Handel, E. und D. B. Zilversmit, J. Clin. Med. 50, 152

(1957). - 3. HaRDING, H. und G. HernzeL, diese Z. 7, 356 (1969).

4. Kessler, G. und H. Lederer, Intern. Technicon Symposium,
Frankfurt (1965). - 5. KLotzsCH, H. und S. KrotzsCH, unpubliziert. - 6. LOFLand, H. B., Analytic. Biochem. 9, 393 (1964). 7. Schmidt, F. H. und K. von DAHL, diese Z. 6, 156 (1968).

Priv.-Doz. Dr. F. H. Schmidt Forschungslaboratorien der Firma Boehringer Mannheim GmbH, (Abt. Stoffwechsel) 6800 Mannheim 31

Sandhofer Straße 112-132 